\title{
Fair Sharing of Bandwidth in VANETs
}

\author{
Marc Torrent-Moreno \\ Institute of Telematics \\ University of Karlsruhe \\ Germany \\ torrent@tm.uni-karlsruhe.de
}

\author{
Paolo Santi \\ Istituto di Informatica e \\ Telematica del CNR \\ Pisa, Italy \\ paolo.santi@iit.cnr.it \\ Technical Report 2005-19 \\ ISSN 1432-7864 \\ Dept. of Computer Science \\ Universität Karlsruhe (TH)
}

\author{
Hannes Hartenstein \\ Institute of Telematics \\ University of Karlsruhe \\ Germany \\ hartenstein@rz.uni-karlsruhe.de
}

\begin{abstract}
We address the challenge of how to share the limited wireless channel capacity for the exchange of safety-related information in a fully deployed vehicular ad hoc network (VANET). In particular, we study the situation that arises when the number of nodes sending periodic safety messages is too high in a specific area. In order to achieve a good performance of safety-related protocols, we propose to limit the load sent to the channel using a strict fairness criterion among the nodes. A formal definition of this problem is presented in terms of a max-min optimization problem with an extra condition of per-node maximality. Furthermore, we propose FPAV, a power control algorithm which finds the optimum transmission range of every node, and formally prove its validity under idealistic conditions. Simulations are performed to visualize the result of FPAV in a couple of road situations. Finally, we discuss the issues that must be taken into account when implementing FPAV.
\end{abstract}

\section{Introduction}

We have witnessed a wide spread of mobile technologies during the last decade. Their rapid evolution and cost reduction have made them to be considered as a suitable solution for a wide spectrum of applications. Recently, the promises of wireless communications to support vehicular safety applications have led to several research projects around the world: the Vehicle Safety Communications Consortium (USA) [1] developing the DSRC technology [2], the Internet ITS Consortium [3] (Japan), the PReVENT project [4] (Europe) or the 'Network on Wheels' project (Germany) [5], to name a few. All these projects have as a main goal to improve safety in vehicular environments by the use of wireless communications, but also consider transport efficiency, comfort and environment. The results achieved so far by the various projects together with the efforts of car manufacturers and standardization bodies, e.g., [6], invite to optimism. Although many problems are not yet solved, the general feeling is that vehicles could benefit from spontaneous wireless communications in a near future, making VANETs (Vehicular Ad-Hoc Networks) a reality. 
In this paper we analyze a problem arising in VANETs with high vehicle densities. In this context, it is likely that the limited capacity of the control channel used to exchange safety-related information is not enough to support the safety-related load generated by a large number of vehicles, unless the offered load is carefully controlled. More specifically, in this paper we consider a fairness problem that arises in situations in which vehicles send periodic beacon messages to inform other vehicles in the surrounding of their current state (velocity, direction, and so on) in order to improve safety conditions. The motivations for studying this problem are thoroughly discussed in Section 2. After presenting our fairness problem and formally defining it in terms of a max-min optimization problem with an extra condition on per-node maximality, we propose an approach to solve this problem based on power control, and we provide an optimal algorithm, called FPAV (Fair Power Adjustment for Vehicular environments) - see Section 3. We then verify the validity of our approach by simulation. The results show FPAV's fairness and effectivness in confining the network load generated by the beaconing activity below a certain desired threshold (Section 4). In Section 5 we discuss the issues that must be dealt with when bringing FPAV into a real scenario. Section 6 presents some related work, and Section 7 concludes the paper.

\section{Motivation for fair power adjustment}

In a VANET every vehicle will be able to send and receive data packets into/from a shared medium. One of the decisions already taken in the USA (FCC ruling report [7]) is that the frequency spectrum will be divided in 7 different channels, 1 control channel and 6 service channels. The control channel will be utilized for the exchange of safety messages, and will contain few service messages, e.g., announcing services, if feasible. Therefore, all vehicles will have to monitor the control channel often enough to receive all safety related information so that the safety applications achieve their goal.

In this paper, we are concerned with the utilization of the control channel. In particular, we assume that two types of safety messages circulate in the control channel and classify them depending on how they are generated: event driven and periodic. The first ones are the result of the detection of an unsafe situation, e.g., a car crash, the proximity of vehicles at high speed, etc. Periodic messages instead can be seen as preventive messages in terms of safety, and their information can also be used by other (non-safety) applications (e.g., traffic monitoring) or protocols (e.g., routing). Periodic message exchange (also called beaconing in the following) is needed to make vehicles aware of their environment. Thus, they will be able to avoid emergency or unsafe situations even before they appear.

We assume, therefore, that beacon messages essentially contain the state of the sending vehicle, i.e., position, direction, speed, etc., and also aggregated data regarding the state of their neighbors. It is reasonable to assume that these periodic messages will be sent in a broadcast fashion since the messages' content can be benefitial for all vehicles around. Finally, it is our strong belief that the amount of load resulting from beaconing should be limited, i.e., the medium should not be working permanently near the maximum load limit. This is because it is desirable to leave some bandwidth available to handle unexpected emergency situations with a reasonable reliability. Emergency packets should be able to access the control channel with short delay, and they should have low probability of collision even when targeting large areas, i.e., when being transmitted with high power. 
In the context described above, a fundamental design decision is to choose a strategy for sending the periodic safety messages. We assume that some communication parameters (e.g., transmission range, packet generation rate) can be appropriately set depending on the situation and/or the vehicles' state. An example of such strategy could be to increase the transmission power of beacons depending on the vehicle's speed. Therefore, we expect different transmission power requirements among the nodes.

When VANETs are fully deployed, they might encounter situations where the technology limitations become a challenge. Scenarios with high vehicle densities can be easily found in real life, e.g., highways at the entrance of big cities or a traffic jam due to a temporal working area. Due to a large number of vehicles sharing the medium, it is not clear whether the channel capacity is sufficient in these scenarios to support the data load generated by beaconing while at the same time leaving enough available bandwidth for event-driven safety messages.

Now, let us consider the following assumptions: $a$ ) the lower layer technology used in VANETs will be a variant of IEEE 802.11a technology [2] and $b$ ) there will be only one control channel, $10 \mathrm{MHz}$ wide [7], for the exchange of both types of safety messages. Carrier Sensing Multiple Access with Collision Avoidance (CSMA/CA), i.e., 802.11 Link Layer protocol, is a totally asynchronous approach. Although it is widely used in commercial applications, it is known for not being able to manage the medium resources very efficiently, especially in case of broadcast messages. Then, a $10 \mathrm{MHz}$ channel can offer half the data-rates of 802.11a, and lower rates are preferred because of their robustness to noise and interference. With such configuration, we conducted a simulation work [8] where broadcast reception rates were evaluated. For instance, we observed that in a scenario with a high node's density and an offered load to the channel $(2.56 \mathrm{Mbps})$ lower than half of the channel's ideal capacity (6Mbps), the probability that a node receives a broadcast message at the edge of the intended communication range ${ }^{1}$ drops below $40 \%$. Basically, the main reason for such low reception rates is the well-known hidden terminal problem (see Figure 1) ${ }^{2}$. Hidden nodes have a severe impact on these scenarios, since no channel reservation process is performed in the targeted area when sending a broadcast packet.

Therefore, we are concerned with situations where the overall load generated by beaconing is too high, i.e., packet collisions are too high, and thus the information obtained by a node cannot be updated frequently enough to prevent possible emergency situations in a vehicle's surrounding. To avoid such situations, we need to design a congestion control mechanism which is able to keep the periodic messages' load under the aforementioned maximum load in all points of the network. This threshold, called MaxBeaconingLoad (MBL) in the following, represents a limit where safety protocols can achieve a reasonable performance. Since MBL represents a network load threshold it is measured in Mbps, however, if we assume a fixed packet generation rate, it might be equivalently measured as maximum number of cars whose CS range cover a specific point in the network, when appropriate.

We propose to adjust the transmission range of all nodes using power control in order to keep the load in the medium below a certain threshold. We are aware that before decreasing the transmission power of safety messages other steps should be taken, for example, implementing an admission control mechanism to drop all non-safety related

\footnotetext{
${ }^{1}$ The intended Communication, or Transmission, Range is the distance up to where a transmission would be received successfully in ideal conditions and in the absence of any interference.

${ }^{2}$ The Carrier Sense range, in ideal conditions, is the distance to which a node's transmissions can be sensed, or in other words, the distance to which a node can interfere with other transmissions.
} 


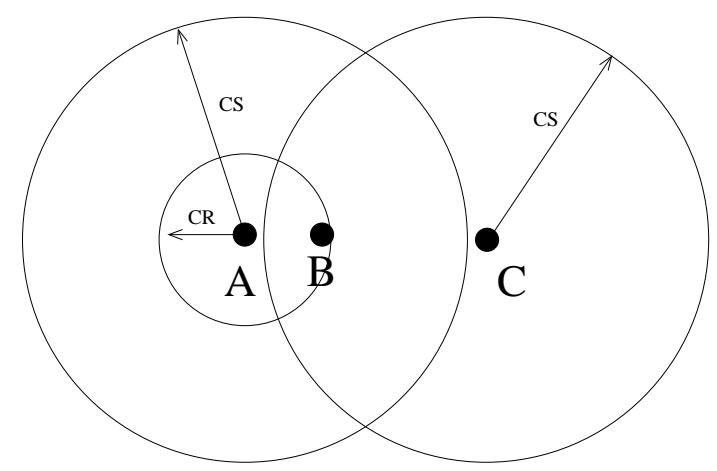

Figure 1: Hidden Terminal Problem: In a CSMA/CA scenario, node B is inside the Communication Range of $\mathrm{A}$ and node $\mathrm{C}$ is placed outside of the Carrier Sense range (CS) of A, i.e., $\mathrm{C}$ can not sense ongoing transmissions from A. In that case, the hidden terminal problem occurs when $\mathrm{B}$ can not receive a message from $\mathrm{A}$ because it collides with one from $\mathrm{C}$.

packets before being sent to the control channel, or minimizing the packet generation rate. Although these strategies can also be utilized as a first step, there will be situations where decreasing the transmission range of certain nodes is necessary. By adjusting the transmission range once the packet generation rate is fixed to the minimum requirement of the safety applications, the load on the channel can be reduced while at the same time high-accuracy information of neighboring vehicles is still available.

Although power control has been a deeply studied subject in the mobile networks field already (see related work in Section 6), vehicular environments present new challenges. As argued above, safety application designers may decide that the beacon's transmission power of a node depends on its state. Since these different power settings should be respected also among neighboring nodes, we introduce the concept of fair power control in VANETs: all vehicles in a certain area must restrict their beacons' (potentially different) transmission power by the same ratio to satisfy MaxBeaconingLoad. Basically, in a high dense cloud of vehicles, our proposal is to decrease the transmission ranges of all nodes by the same ratio until there is no spatial area where the load overcomes the pre-fixed maximum $M B L$. We present in the following section a detailed and formal definition.

\section{The FPAV Algorithm}

\subsection{The reference application scenario}

We are considering a scenario in which a set of vehicles (also called cars, users, or nodes, in the following) is moving along a road. Periodically, users send beacon messages to inform the nodes in their vicinity of their current position, direction, velocity, etc. We assume that the beaconing frequency is the same for all the nodes in the network. However, the power used to transmit beacons can be adjusted, so that the overall network bandwidth used for beaconing can be kept under control.

In principle, a node will send its beacon at maximum power, as this in general guarantees that more nodes will receive the beacon, resulting in increased safety conditions. On the other hand, the higher the power used to send beacons, the higher is the network load generated by the beacon exchange activity. 
We recall that in the envisioned application scenario, the above described beaconing activity is assigned with a limited portion of the available network bandwidth, the remaining bandwidth being available for event-driven safety messages. Thus, the 'node optimal strategy' of sending the beacon at maximum power in general conflicts with the network-wide task of keeping the network load offered by beaconing below a certain threshold. As a consequence of this, we need a strategy for setting the node transmit power levels, such that the beaconing network load does not exceed the threshold, and the beaconing transmit power levels are maximized.

\subsection{The BMMTxP problem}

Assume a set of nodes $N=\left\{u_{1}, \ldots, u_{n}\right\}$ is moving along a road. To simplify the problem statement, we assume that the road is modeled as a line ${ }^{3}$ of unit length, i.e. $R=[0,1]$, and that nodes can be modeled as points in $[0,1]$. Given a node $u_{i} \in$ $N, x(i, t)$ denotes the position of $u_{i}$ in $R$ at time $t$. To simplify the notation, in the following we drop the argument $t$, focusing our attention on a snapshot of the system at a certain time instant $t$. Mobility is later addressed in Section 5.

Each of the network nodes sends a beacon with a predefined beaconing frequency $F$, using a certain transmit power $p \in\left[0, P_{\max }\right]$, where $P_{\max }$ is the maximum transmit power. In order to simplify the presentation, we assume that all the nodes have the same maximum transmit power level. We remark that this assumption is made only to simplify the notation, and that the framework described in this paper can be applied also when the nodes have different maximum transmit power levels.

Definition 1 (Power assignment) Given a set of nodes $N=\left\{u_{1}, \ldots, u_{n}\right\}$, a power assignment $P A$ is a function that assigns to every network node $u_{i}$, with $i=1, \ldots, n$, a ratio $P A(i) \in[0,1]$. The power used by node $u_{i}$ to send the beacon is $P A(i) \cdot P_{\max }$.

Definition 2 (Interference Range) Given a power assignment $P A$ and any node $u_{i} \in$ $N$, the interference range of $u_{i}$ under $P A$, denoted $I R(i, P A)$ is defined as the intersection between the $C S$ range of node $u_{i}$ at power $P A(i) \cdot P_{\max }$ and the deployment region $R$.

The above definition of interference deserves some explanation. In general, assuming that the CS range can be modeled as a $1 / 0$ situation (either a transmission at a certain power interferes with a node, or it does not interfere at all) is a simplification of what occurs in practice, where the wireless channel conditions (which have a strong influence on the quality of the received signal) fluctuate over time. It is not difficult to extend our definition of interference to account for variable channel conditions: essentially, it is sufficient to associate a certain probability density function over $[0,1]$ to each pair $\left(u_{i}, P A(i)\right)$. However, in order to simplify the presentation of our framework, we assume that the notion of interference range is deterministic.

Besides the 0/1 interference assumption described above our notion of interference range is very general, as we do not assume that the CS range is regular - e.g. a segment centered at $x(i)-$, nor that it is contiguous - due to the presence of obstacles, there might exist 'holes' in the interference region. The only other assumption which is needed for the correctness of the proposed framework is a monotonic property, namely

\footnotetext{
${ }^{3}$ Modeling the road as a line is a reasonable simplification in our case since we assume the communication ranges of the nodes to be much larger than the width of the road.
} 


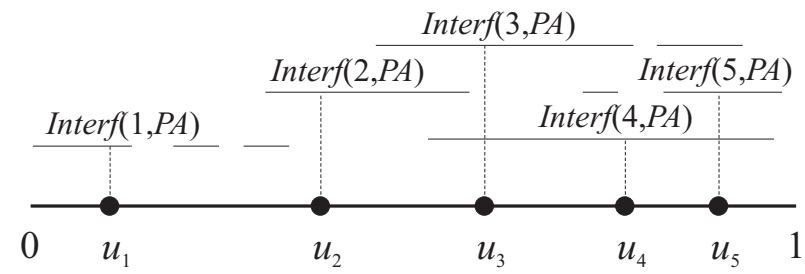

Figure 2: Network load based on interference: the maximum load is experienced in those subregions of $R=[0,1]$ where the number of intersecting interference ranges is maximal. In the example, we have $B L(P A)=3$.

that the interference range of node $u_{i}$ at power $(P A(i)+\epsilon) \cdot P_{\max }$ contains the interference range of node $i$ at power $P A(i) \cdot P_{\max }$, for every $\epsilon>0$. We remark that this assumption is very reasonable in a realistic setting.

Given a power assignment $P A$, the network load generated by the beaconing activity under $P A$ is defined as follows:

Definition 3 (Beaconing load under $P A$ ) Given a set of nodes $N$ and a power assignment $P A$ for the nodes in $N$, the beaconing network load under $P A$ is defined as

$$
B L(P A)=\max _{x \in[0,1]} \text { Interference }(x, P A),
$$

where Interference $(x, P A)$ is the number of nodes which have point $x$ in their $C S$ range under $P A$. Formally,

$$
\text { Interference }(x, P A)=\left|\left\{u_{i} \in N: x \in I R(i, P A)\right\}\right| \text {. }
$$

An example clarifying our notion of network load based on interference is reported in Figure 2. The intuition is the following: since the beaconing frequency is predetermined, the network load depends on the transmit power levels used for beaconing - the higher these levels, the higher the network load ${ }^{4}$. The maximum load is experienced in those subregions of $R$ where the number of intersecting interference ranges is maximal.

We are now ready to define the beaconing with max-min transmit power problem addressed in this paper:

Definition 4 (Beaconing Max-Min Tx power Problem (BMMTxP)) Given a set of nodes $N=\left\{u_{1}, \ldots, u_{n}\right\}$ in $R=[0,1]$, determine a power assignment $\overline{P A}$ such that the minimum of the transmit powers used by nodes for beaconing is maximized, and the network load remains below the beaconing threshold MBL. Formally,

$$
\left\{\begin{array}{l}
\max _{P A \in \mathbf{P A}}\left(\min _{u_{i} \in N} P A(i)\right) \\
\text { subject to } \\
B L(P A) \leq M B L
\end{array}\right.
$$

where PA is the set of all possible power assignments.

Informally speaking, we are interested in finding the power assignment such that the minimal QoS guaranteed to the network nodes is maximized, i.e., is fair to all

\footnotetext{
${ }^{4}$ Here, we use the assumption of monotonic interference range.
} 
nodes, while not exceeding the portion of network bandwidth assigned to the beaconing activity. Notice that in our problem formulation we are assuming that the portion of bandwidth assigned for beaconing can be expressed in terms of the maximal overlapping between the nodes' interference ranges. This assumption is reasonable under our working hypothesis of fixed beaconing frequency.

Observe that in general there exist several power assignments that can be regarded as optimal solutions to BMMTXP. For instance, assume a certain power assignment $\overline{P A}$ is optimal for BMMTxP, and assume there exists a node $u_{i} \in N$ such that the power assignment $\overline{P A}(i, \epsilon)$ obtained from $\overline{P A}$ by increasing $u_{i}$ 's transmit power to $(P A(i)+\epsilon) \cdot P_{\max }$, for some $\epsilon>0$, does not violate the condition on the network load. It is immediate to see that also $\overline{P A}(i, \epsilon)$ is an optimal solution to BMMTXP.

In general, we are interested in finding an optimal solution to BMMTxP which is per-node maximal, i.e. a power assignment $\overline{P A}_{M}$ such that increasing the transmit power of any single network node results in exceeding the assigned network bandwidth.

Definition 5 (Per-node maximal power assignment) A power assignment $\overline{P A}_{M}$ for node set $N=\left\{u_{1}, \ldots, u_{n}\right\}$ is per-node maximal if and only if:

i) it is an optimal solution to BMMTXP; and

ii) for each $u_{i} \in N$, and for any $\epsilon>0$, we have that $B L\left(\overline{P A}_{M}(i, \epsilon)\right)>M B L$.

Our interest in finding a per-node maximal power assignment is motivated by the fact that, as long as the condition on the network load is not impaired and the minimum of the nodes' transmit power levels is maximized, the higher a node transmit power the better the safety conditions of the vehicle.

\subsection{An optimal algorithm for BMMTxP}

In this section we present a centralized algorithm for solving BMMTxP and computing a per-node maximal power assignment. The algorithm, called FPAV (Fair Power Adjustment for Vehicular environments), is composed of two stages: stage 1 computes an optimal solution to BMMTxP, and stage 2 augments this solution into a per-node maximal power assignment.

Stage 1 of FPAV, which is summarized in Figure 3, is very simple: every node starts with the minimum transmit power, and all the nodes increase their transmit power simultaneously of the same amount $\epsilon \cdot P_{\max }$ as long as the condition on the beaconing network load is satisfied.

The next theorem shows that this simple strategy results in producing an optimal solution to BMMTxP. Technically, the power assignment computed by stage 1 of FPAV is an $\epsilon \cdot P_{\text {max }}$-approximation of the optimal solution to BMMTxP. Since the step size $\epsilon$ is an arbitrarily small constant, the solution computed by BMMTxP can be regarded as optimal for all practical purposes.

Theorem 1 Stage 1 of FPAV computes an $\epsilon \cdot P_{\max }$-approximation of the optimal solution to BMMTxP for any constant $\epsilon>0$.

Proof: First, we observe that the power assignment $P A$ computed by the stage 1 of FPAV, with a power level $p=(k \epsilon) \cdot P_{\max }$, is the minimal assignment among all the power assignments with minimum power level $p$, since in $P A$ all the nodes have the same power level $p$. Thus, if a power assignment $P A^{\prime}$ with minimum power level 
Algorithm FAN, STAGE 1:

INPUT: $\quad$ a set of nodes $N=\left\{u_{1}, \ldots, u_{n}\right\}$ in $[0,1]$

OUTPUT: $\quad$ a power assignment $P A$ which is an ( $\epsilon$-approximation of an) optimal solution to BMMTxP

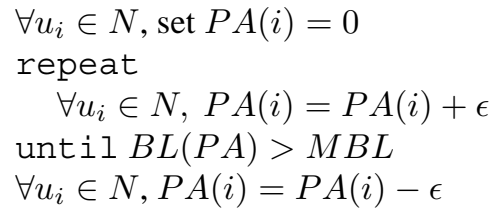

Figure 3: Stage 1 of the FAN algorithm.

$p$ does not violate the condition on the network load, then also $P A$ does not violate the condition on the network load because the nodes' interference ranges under $P A^{\prime}$ are at least as large as under $P A$ (this is true because of the assumption of monotonic interference range).

Let $\bar{p}$ be the minimum of the node transmit powers in an optimal solution to BMMTxP, and assume $(k \epsilon) \cdot P_{\max }<\bar{p} \leq((k+1) \epsilon) \cdot P_{\max }$ for some $k \geq 0$. The following cases can occur:

(i) $\bar{p}=((k+1) \epsilon) \cdot P_{\max }$. In this case, given the observation above it follows immediately that the power assignment computed by FPAV-stage 1 is optimal;

(ii) $(k \epsilon) \cdot P_{\max }<\bar{p}<((k+1) \epsilon) \cdot P_{\max }$. In this case, given the observation above and the assumption of monotonic interference range we can conclude that the power assignment $P A$ computed by FPAV-stage 1 is a feasible solution to $B M M T x P$, which is at most $\epsilon \cdot P_{\max }$ away from the optimal solution.

This concludes the proof of the theorem.

Observe that we had to introduce the constant $\epsilon$ in our algorithm to discretize the process of increasing the nodes' transmit power. The smaller $\epsilon$, the more accurate the solution computed by FPAV, the longer the running time of the algorithm. On the other hand, in a practical setting we expect that nodes can set the transmit power only to a limited number of different levels, and discretizing the transmit power increase process is not an issue. It is immediate to see that, under the assumption that all the nodes use the same power levels $\left\{p_{1}, \ldots, p_{h}\right\}$, stage 1 of FPAV computes an optimal solution to BMMTxP (subject to the constraint that the possible power levels for the nodes are $\left.\left\{p_{1}, \ldots, p_{h}\right\}\right)$.

Let use now consider stage 2 of FPAV, which is summarized in Figure 4. Also this stage is very simple: given an optimal solution to $B M M T x P$ (provided by stage 1 of the algorithm), each node is considered in turn, and its transmit power is increased by $\epsilon \cdot P_{\max }$ as long as the condition on the beaconing network load is satisfied.

The next theorem shows that stage 2 of FPAV computes a per-node maximal (technically, an $\epsilon \cdot P_{\max }$-approximation to a per-node maximal) power assignment.

Theorem 2 Assume PA is an optimal solution to BMMTxP; then, stage 2 of FPAV computes an $\epsilon \cdot P_{\text {max }}$-approximation to a per-node maximal power assignment for any constant $\epsilon>0$. 
AlgORITHM FAN, STAGE 2:

INPUT: $\quad$ an optimal solution to BMMTxP, denoted $P A$

OUtPUT: a power assignment $P A$ which is a ( $\epsilon$-approximation of a) per-node maximal power assignment

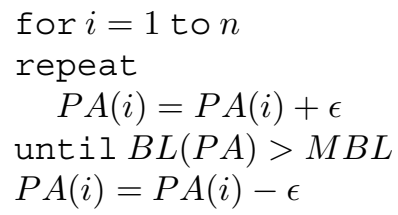

Figure 4: Stage 2 of the FAN algorithm.

Proof: The proof is along the same lines as the proof of Theorem 1. Let $P A$ be an optimal solution to BMMTxP; this power assignment is augmented by FPAV-stage 2 considering each node $u_{i}$ in turn, which is assigned with a power level that is at $\operatorname{most} \epsilon \cdot P_{\max }$ away from the maximal power level for node $u_{i}$ which does not violate the condition on the network load. It follows that the power assignment computed by FPAV-stage 2 at the end of this augmentation process is such that the power assigned to each node is at most $\epsilon \cdot P_{\max }$ away from the per-node maximal transmit power level. We can then conclude that FPAV-stage 2 computes an $\epsilon \cdot P_{\max }$-approximation to a per-node maximal power assignment.

For reasons similar to the ones discussed above, the power assignment computed by FPAV-stage 2 can be regarded as per-node maximal for all practical purposes.

\section{Experiments}

To illustrate the performance of FPAV and as a 'proof of concept' we have implemented the algorithm in $\mathrm{C}$ and simulated it under different traffic situations.

Since many decisions regarding the technology to be used in VANETS are not yet taken, we are forced to do additional assumptions or approximations. To define the load that every node periodically intends to offer to the control channel we should fix two parameters (the third parameter, transmit power, is managed by FPAV): packet generation rate and packet size. We assume that broadcasting a few packets per second is sufficient to maintain an accurate knowledge of position and state of all cars. On top of that, the number of transmitted packets may be increased due to retransmissions or to the use of mechanisms for improving transmission reliability. We take 10 packets per second as a reasonable rate for periodic messages. To come up with a packet size value we consider that every packet will contain several parameters composing the state of the sender. Also, the beacon could contain some aggregated and very valuable information about the sender's neighbors. If we finally consider some necessary security fields it does not look too pessimistic to take 250 Bytes as the packet size. These two parameters set the offered load of every node to $20 \mathrm{Kbps}$ inside their $\mathrm{CS}$ range. To facilitate interpretation of the results we fix the same intended communication range for all nodes, i.e., we assume that the radio coverage area is regular (no holes), and that the maximum CR is $250 \mathrm{~m}$. Similarly, we assume that the interference range of a 
node has a regular shape (no holes). Also, a maximum load accepted for the control channel should be defined. Taking into consideration all arguments from Section 2 we set the maximum load for beaconing to $50 \%$ of the channel capacity. For the physical layer we choose one of the lower 802.11 a rates, $6 \mathrm{Mbps}$, since this rate is more robust against interferences, i.e., nodes will have a shorter CS minimizing the effect of hidden terminals. Then, assuming a required SNR of $6 \mathrm{~dB}$ and that idealistically the power decreases with the square of the distance we will have a CS of $500 \mathrm{~m}$ approximately (at maximum transmit power). The final parameter we have to fix is the resolution of $P A$ 's increase in FPAV, i.e., the step size $\epsilon$. We fix this parameter to 0.01, resulting in a CS increase of 5 meters for each increase of $P A$.

On the other hand, we also have to specify a vehicular traffic scenario to run our simulations. We choose a straight linear road with an average vehicle density of 20 vehicles per $100 \mathrm{~m}$ modeling a congested traffic situation ${ }^{5}$. We recall that even higher vehicle densities can be easily found every day on real roads. Finally, we must consider that in most situations both directions of the traffic will share the same communication medium.

A summary of the configuration parameters of our simulations can be found in Table 1.

\begin{tabular}{|l|l|}
\hline Packet generation rate & 10 pckts/s \\
\hline Packet size & $250 \mathrm{~B}$ \\
\hline Load $_{\text {vehicle }}$ & $20 \mathrm{Kbps}$ \\
\hline Data Rate & $6 \mathrm{Mbps}$ \\
\hline Maximum beaconing load & $3 \mathrm{Mbps}$ \\
\hline Communication range & $250 \mathrm{~m}$ \\
\hline Carrier Sense range & $500 \mathrm{~m}$ \\
\hline Step size $\epsilon$ & 0.01 \\
\hline Vehicle density & $20 \mathrm{vehicles} / 100 \mathrm{~m}$ \\
\hline
\end{tabular}

Table 1: Configuration parameters

Let us now define the metrics used to evaluate FPAV performance:

- Offered Load: Load, accumulated from all nodes, offered to the control channel [Mbps] in a specific point $x$ on the road before applying FPAV. This metric shows the resulting load offered to the channel if no power control is performed (i.e., all vehicles have $P A(i)=1$ ).

- Adjusted Load: Load, accumulated from all nodes, offered to the control channel [Mbps] in a specific point $x$ after all nodes have adjusted their transmit power according to FPAV.

- MaxBeaconingLoad (MBL): Maximum load allowed for beaconing [Mbps].

- $P A$ value: Value of $P A(i)$ after FPAV execution, expressed as a function of the node position $x$. Note that all vehicles placed on the same position $x$ (if any) will have the same value of $P A$ due to the same configuration values.

\footnotetext{
${ }^{5}$ As in Section 3 we model the road as a line (1-D). Thus, our densities will be given in [vehicles/m] instead of [vehicles $/ \mathrm{m}^{2}$ ] adding up all vehicles circulating in the different lanes. For example, in a 4 lanes road, to have 20 vehicles $/ 100 \mathrm{~m}$ results in 1 car every $25 \mathrm{~m}$ in each lane.
} 


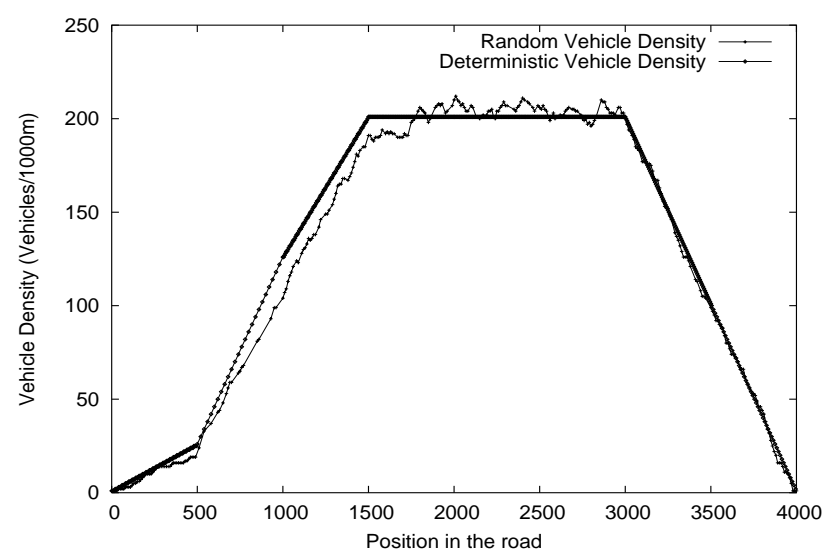

Figure 5: Traffic Cloud Densities. Vehicle densities at each point for both deterministic and random scenarios.

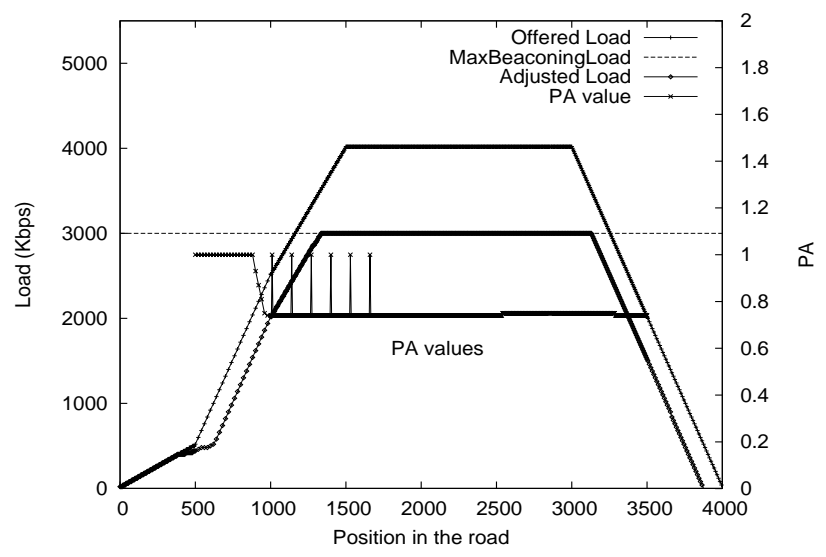

Figure 6: Deterministic Traffic Cloud. Load on the channel at every point of the deterministic scenario before and after applying FPAV.

- Vehicle Density: Number of vehicles in a range of $1000 \mathrm{~m}$ [vehicles $/ 1000 \mathrm{~m}$ ] centered in a specific point $x .1000 \mathrm{~m}$ is selected to give an estimation of the number of cars a node has inside its maximum CS (when $P A=1$ ).

We present the results obtained from applying FPAV to two different traffic scenarios. Both of them model the same piece of road (4km long) and have the same overall car density. However, in the first one cars are placed in a deterministic, equally-spaced fashion (Deterministic Vehicle Density Fig. 5). On the other hand, in the second scenario, vehicles are placed somehow randomly (Random Vehicle Density Fig. 5). In the following, we will refer them as deterministic or random scenario, respectively. In order to facilitate presentation and comprehension of results, both scenarios model a static situation.

The deterministic scenario models a cloud of cars in a straight road (starting at $x=500 \mathrm{~m}$ ) where the first 0.5 kilometer ('rear part' of the cloud) is populated with 1 car every 20 meters and the following 2.5 kilometers with 1 car every 5 meters ('front 
part' of the cloud). To populate the second scenario we make use of a discrete uniform random number generator. In particular, we place every $10 \mathrm{~m}$ either 0 or 1 car along the first $0.5 \mathrm{~km}$, and a number of cars ranging between 0 and 4 along the following 2.5 kms. Notice that in the plot reported in Figure 5 we do not report the parameters' values if at a certain point $x$ there are no cars. This explains the missing points, e.g., around $x=800 m$ in the random scenario curve due to the result of the random generator being 0 in that point.

Figure 6 provides some insight into how FPAV works. The original Offered-Load, which exceeds $M B L$, has been adjusted right below this threshold. The values of $P A$ at the end of FPAV's execution look fairly distributed. However, we can appreciate how few nodes are allowed to transmit with much higher power than their neighbors since stage 2 of FPAV has not strict fairness constraints as stage 1. Let us now take a closer look to Figure 6 to better understand FPAV's behavior. First, we notice that curves in Figure 6 represent the result from the car distribution plotted as Uniform Vehicle Density (Fig. 5). Since we computed the Vehicle Density over a range of 1000m, the Offered Load matches Figure 5 scaled up by a factor of $20 \mathrm{Kbps} / \mathrm{car}$ (this also applies for the random case). Now, we can observe how the channel load increases with the car density in the rear side of the cloud (lower $x \mathrm{~s}$ ). At the same time, the increase of the vehicle density causes the $P A$ values to start decreasing (at $x=840 \mathrm{~m}$ ). At this point too many vehicles intend to transmit inside this specific region, and the value of $P A$ decreases from 1 (where density is still $1 \mathrm{car} / 20 \mathrm{~m}$ ) to 0.75 (where density starts being $1 \mathrm{car} / 5 \mathrm{~m})$. Then, the values of $P A$ remain constant ( 074 or 0.75 except for the few nodes commented above) up to the first car of the cloud $(x=3500 \mathrm{~m})$. The value of $P A$ as computed at the end of the first stage of FPAV in the denser region of the road can be easily calculated as follows:

$$
P A=\frac{\text { MaxBeaconingLoad }}{2 * C S_{\max } * \text { Vehicle_Density } * \text { Load }_{\text {vehicle }}}-\epsilon
$$

Note that if we would not subtract $\epsilon$ from $P A$ the resulting load would exceed MaxBeaconingLoad since in our discrete scenario in a range of, e.g., 500m there are 101 cars and not 100. Thus:

$$
P A=\frac{3 M b p s}{2 * 500 m * 1 c a r / 5 m * 20 K b p s / c a r}-0.01=0.74
$$

The reason for $P A=0.75$ in some regions of the road, i.e., between $x=2540 \mathrm{~m}$ and $x=3285 \mathrm{~m}$, and $P A=1$ for few nodes between $x=1010 \mathrm{~m}$ and $x=1660 \mathrm{~m}$ is that $P A=0.74$ is not a per-node maximal solution. Therefore, some nodes can increase their transmission power (in turn) without violating the condition on the network load $B L(P A) \leq M B L$.

Very interesting are also the results obtained from the random scenario (Fig. 7). Observe how FPAV achieves a good channel utilization, i.e., the Adjusted Load stays very close to the $M B L$ whenever possible. Contrary to the previous scenario and due to the random distribution of the nodes, cars in the front part of the cloud do not in general have the same value of $P A$ after executing FPAV, as we would expect from a real traffic situation. 


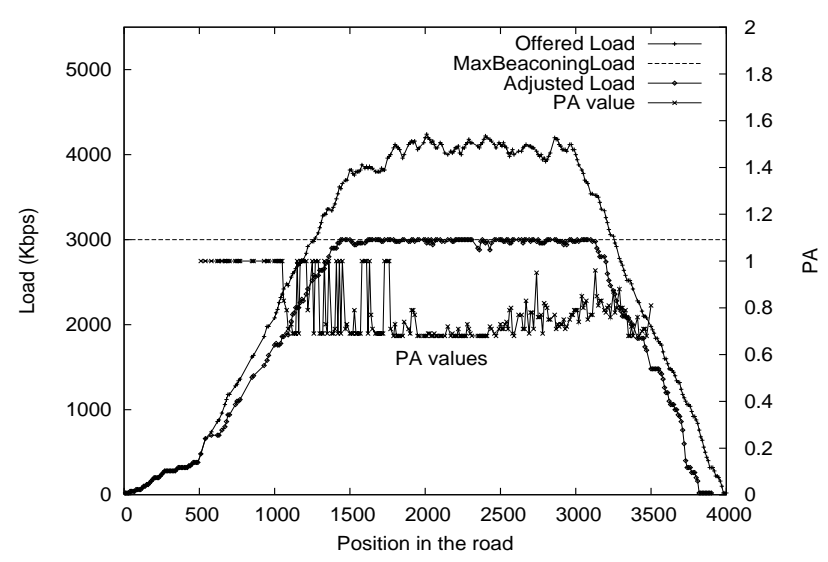

Figure 7: Random Traffic Cloud. Load on the channel at every point of the random scenario before and after applying FPAV.

\section{Discussion}

We have presented a power control approach that achieves fairness when adjusting the channel load in VANET environments. In this section, we discuss the feasibility of our approach and outline open issues we have to consider when specifying an implementation for real environments.

Determining the MaxBeaconingLoad threshold. The determination of the MaxBeaconingLoad threshold depends on safety requirements of the applications that must be fixed by application designers. We expect that, in order for the safety system to be reliable, safety applications should be updated with new information $a$ ) within a specific time and $b$ ) with some minimum reliability. Hence, issues to consider when determining the MaxBeaconingLoad are the accuracy of the positioning devices, the reaction time of the potential drivers, the efficiency of the breaking system, etc. Also, a very important issue to address is how to balance the accuracy and amount of information. The trade-off situations spans between using low transmission power for accurate state information only from nearby nodes to using high transmission power to acquire less accurate state information from more nodes including further ones.

Once safety related issues are addressed, communication challenges come into play. Note that, as commented in Section 3, we assume a constant packet generation rate for all cars performing beaconing. Nevertheless, accurately estimating the maximum load in the channel that guarantees a minimum performance level is not straightforward in our environments. In fact, wireless medium access control protocols have to deal with the hidden terminal problem, with non-deterministic channel characteristics, and mobility. Therefore, a thorough study of this issue will be needed when technology requirements will be defined.

Implementation issues. Our goal is to implement FPAV in a fully distributed, localized and asynchronous fashion. Note that the current version of FPAV is centralized and requires synchronization between nodes (especially Stage 1). In principle, we can think about two approaches to solve this problem. The first one is a mere distributed implementation of FPAV, i.e., the same protocol is executed at each node, and nodes increase their $P A$ value synchronously until the MaxBeaconingLoad threshold is reached. Implementing this approach would require a tight synchronization among the nodes and 
a 'global knowledge' of the channel load to determine whether the condition on the maximum allowed load is satisfied in the entire network. We believe this approach is not feasible in VANETs where the high degree of mobility renders the goals of ensuring tight synchronization and of quickly assessing global information too ambitious (at least with current technology).

The second approach is to let every node execute FPAV 'internally' (i.e., without synchronization and network-wide check of maximum offered load), assuming nodes have some knowledge about their environment. Observe that, if nodes would know the exact position of the other nodes and the channel characteristics (e.g., their CS range), they could run FPAV by themselves and compute the resulting $P A$ values of all nodes for that specific situation, and this set of values would be the same for all the nodes (assuming all the nodes have the same knowledge). Clearly, the performance of this approach depends on how accurate the knowledge about the state of other vehicles is, and whether the nodes have complete knowledge of the environment (as it is assumed above), or only a partial knowledge of the environment. In the latter case, the computed solution (i.e., setting of the $P A$ values) would probably be sub-optimal, but the induced load needed to maintain environment information would be minimized. A careful study of this approach, and of the tradeoff between computing an optimal solution with global knowledge (but high overhead to maintain the environment information) and computing a suboptimal solution with only local knowledge is left to future work.

Real channel characteristics. The unreliability of the wireless channel due to, e.g., fading, will affect the accuracy of the state information acquired from the other vehicles on the road. In reality, the probability that a packet is successfully received does not only depend on collisions but also on the SINR (Signal to Interference and Noise Ratio). In absence of collisions, the higher the received power the lower the BER (Bit Error Rate), see for example the curves that Yin et al. provide in [9]. The trade-off between lower BER and higher interferences must be taken into account when designing any wireless system, specially in VANET's environments, i.e., in highly populated broadcast scenarios.

\section{Related Work}

Channel capacity and power control are broadly studied concepts in ad hoc networks. We can find studies since the early years, Kleinrock and Tobagi [10] analyze the throughput of CSMA transmission protocols already in 1975. Since then a large number of studies tried to optimize the channel throughput or capacity adjusting the transmission power. Up to now, though, no study addressed our specific situation. The particularity of having safety as main goal brings to VANETs new constraints not considered before. Most of the studies address unicast environments and try to improve the spatial reuse minimizing the interference or energy consumption. These studies find the path to the destination that minimizes energy consumption and/or maximizes the overall throughput. In the category of 'energy concerned protocols' would fit most of the topology control proposals such as [11], [12] and [13] that propose adaptive algorithms that make use of only local information to adjust their power or [14] that considers non-uniform transmission ranges. A slightly different approach is given in [15], [16] and [17] where the authors agree that the minimum transmission power does not always maximize throughput and then propose an adaptive algorithm as a function of the traffic load. Although we can find related issues and methodologies in all 
these works we have to remember that energy efficiency is not an issue in VANETs where nodes have unlimited power supply. In addition, another common goal of these approaches is to keep the network connected for unicast flows, which is a totally different approach than the one we are considering. For FPAV the goal is to make sure that nodes close to the sender will receive its messages with high probability while ensuring fairness in the overall system.

Maybe the most related piece of work to our study is performed by Li et al. in two steps [18] and [19]. The authors propose, first, an analytical model able to find a transmission power that maximizes 1-hop broadcast coverage and, second, an adaptive algorithm that converges to the beforehand fixed transmission power. Although they focus on a pure broadcast environment their assumptions make their approach infeasible for VANETs: $a$ ) all nodes are static and $b$ ) all nodes use the same transmission power.

Last, early this year appeared a study [20] where the effect of power control is identified in many wireless parameters. Although they had also in mind the before mentioned classical wireless networks goals, i.e., energy consumption, connectivity and throughput, their explanations can help understand some of the situations considered in former sections.

\section{Conclusions}

In this paper we studied a problem that VANETs will face when achieving high penetration rates in dense roads, i.e., the limited channel capacity to support the exchange of safety-related information. In these scenarios we consider that all nodes can send two types of safety related messages: $a$ ) periodic messages to make the other cars aware about their state and $b$ ) emergency messages triggered by the detection of a non-safe situation. In order to ensure that both types of messages can be handled efficiently with the existent resources we propose to limit the wireless channel load resulting from the periodic messages. Moreover, we require a strict fairness among the vehicles because of the safety nature of VANET applications.

With the constraints commented above, and assuming a constant packet generation rate, we formally defined the challenge in terms of a max-min optimization problem and extend it to obtain per-node maximality. Additionally, we proposed FPAV, a centralized power control algorithm that provides an optimal solution to the defined problem in two stages. In stage 1 FPAV maximizes the minimum transmission range for all nodes in a synchronized approach. In stage 2 FPAV achieves a maximum transmission range for all nodes individually while satisfying the condition of keeping the channel load under a certain limit. We proved the validity of FPAV formally and visualize its performance with simulations under idealized conditions. Finally we discussed all issues that will have to be dealt with when bringing the algorithm into a real scenario: a) finding the proper maximum load threshold that ensures a good performance of the safety protocols, $b$ ) optimizing the performance of the algorithm with only local information and $c$ ) fighting against the adverse and uncertain wireless channel conditions. In our future work we will perform a detailed study of these issues. Our goal is to come up with a fully distributed, localized and asynchronous implementation of the protocol and to validate its performance comparing it with the optimum computed by FPAV as defined in this paper. 


\section{Acknowledgments}

The authors would like to thank Prof. Raja Sengupta and his team at U.C. Berkeley, Qing Xu, Tony Mak, Jeff Ko and Anthony Patire, for useful and stimulating discussions on earlier versions of this paper.

Marc Torrent-Moreno acknowledges the support of the German Ministry of Education and Research $(\mathrm{BMB}+\mathrm{F})$ within the "Network-on-Wheels" project under contract number 01AK064F.

\section{References}

[1] "Vehicle Safety Communications Consortium," http://www-nrd.nhtsa.dot.gov/pdf/nrd-12/CAMP3/pages/VSCC.htm.

[2] "Dedicated Short Range Communications Project," http://www.leearmstrong.com/DSRC/DSRCHomeset.htm.

[3] “Internet ITS Consortium," http://www.internetits.org.

[4] “The PReVENT Project," http://www.prevent-ip.org.

[5] “The NOW: Network on Wheels Project," http://www.network-on-wheels.de.

[6] "Dedicated Short Range Communications working group," http://grouper.ieee.org/groups/scc32/dsrc/index.html.

[7] "Federal Communications Commision. FCC 03-324. FCC Report and Order," February 2004.

[8] M. Torrent-Moreno, D. Jiang, and H. Hartenstein, "Broadcast Reception Rates and Effects of Priority Access in 802.11-Based Vehicular Ad-Hoc Networks," in Proceedings of ACM International Workshop on Vehicular Ad Hoc Networks, Philadelphia, Pennsylvania, October 2004.

[9] J. Yin, T. ElBatt, G. Yeung, B. Ryu, S. Habermas, H. Krishnan, and T. Talty, "Performance Evaluation of Safety Applications over DSRC Vehicular Ad Hoc Networks," in Proceedings of ACM International Workshop on Vehicular Ad Hoc Networks, Philadelphia, Pennsylvania, October 2004.

[10] L. Kleinrock and F. Tobagi, "Packet Switching in Radio Channels: Part I-Carrier Sense Multiple-Access Modes and Their Throughput-Delay Characteristics," IEEE Transactions on Communications, December 1975.

[11] Y. Chen, E. Sirer, and S. Wicker, "On Selection of Optimal Transmission Power for Ad hoc Networks," in Proceedings of Hawaii International Conference on System Sciences (HICSS), Waikoloa, Hawaii, January 2003.

[12] M. Kubisch, H. Karl, A. Wolisz, L. Zhong, and J. Rabay, "Distributed Algorithms for Transmission Power Control in Wireless Sensor Networks," in Proceedings of IEEE Wireless Communications and Networking (WCNC), March 2003. 
[13] X. Chen, M. Faloutsos, and S. Krishnamurthy, "Power Adaptive Broadcasting with Local Information in Ad hoc Networks," in Proceedings of the International Conference on Network Protocols (ICNP), Atlanta, Georgia, November 2003.

[14] W. S. X. Li and Y. Wang, "Efficient Topology Control for Wireless Ad Hoc Networks with Non-uniform Transmission Ranges," in Proceedings of ACM Wireless Networks (WINET), 2003.

[15] S. Park and R. Sivakumar, "Quantitative Analysis of Transmission Power Control in Wireless Ad-hoc Networks," in Proceedings of International Conference on Parallel Processing Workshops (ICPP), Vancouver, Canada, August 2002.

[16] S. Park and R. Sivakumar, "MobiHoc Poster: Adaptive Topology Control for Wireless Ad hoc Networks," ACM SIGMOBILE Mobile Computing and Communications Review (MC2R), July 2003.

[17] J. Liu and B. Li, "MobileGrid: Capacity-aware Topology Control in Mobile Ad Hoc Networks," in Proceedings of IEEE International Conference on Computer Communications and Networks (ICCCN), Miami, Florida, October 2002.

[18] X. Li, T. Nguyen, and R. Martin, “An Analytic Model Predicting the Optimal Range for Maximizing 1-HopBroadcast Coverage in Dense Wireless Networks," in Proceedings of International Conference on Ad-Hoc Networks and Wireless (ADHOC-NOW), Vancouver, Canada, July 2004.

[19] X. Li, T. Nguyen, and R. Martin, “ Using Adaptive Range Control to Maximize 1-Hop Broadcast Coverage in Dense Wireless Networks," in Proceedings of IEEE International Conference on Sensor and Ad hoc Communications and Networks (SECON), Santa Clara, CA, October 2004.

[20] V. Kawadia and P. Kumar, "Principles and Protocols for Power Control in Wireless Ad Hoc Networks," IEEE Journal on Selected Areas in Communications (JSAC), January 2005. 